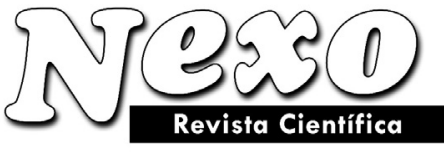

\title{
Fórmulas de recurrencia entre Pm y la derivada k-esima de la delta de Dirac soportada en $\mathbf{P}^{*}$
}

\author{
M. Aguirre. \\ Núcleo Consolidado Matemática Pura y Aplicada-NUCOMPA \\ Facultad de Ciencias Exactas UNCentro, Pinto 399, \\ 7000 Tandil, Argentina. \\ e-mail: $\underline{\text { maguirre@exa.unicen.edu.ar }}$
}

(recibido/received: 08-Agosto-2009; aceptado/accepted: 05-Diciembre-2009)

RESUMEN

En este artículo se le dio un sentido a la fórmula de recurrencia $P^{m} \cdot \delta^{(k)}(P)-C m, k \delta^{(k-m)}(P)=0$ si $k \geq m$ (ver fórmula 15) considerando la condición $\operatorname{grad} P \neq 0$, donde la constante $C m, k$ fue definida por la fórmula 16 . En el segundo parágrafo se le dio un sentido a la misma fórmula pero para un caso especial: $P=P(x)=P\left(x_{1}, \ldots x_{n}\right)=x_{1}^{2}+x_{2}^{2}+\ldots x_{p}^{2}-x_{p+1}^{2}-\ldots x_{p+q}^{2}$.

La fórmula que se obtuvo es una generalización de fórmulas que aparecen en el libro de Gelfand and Shilov formula (c.f. ([1]), página 233) y es considerada por ejemplo por Bollini, Giambiagi and Tiomno para la teoría de regularización analítica en las ecuaciones clásicas de Yang-Mills y sus aplicaciones para el potencial singular (c.f. [4]).

Palabras Claves: Recurrencia; potencia singular

\section{ABSTRACT}

In this paper we gave a sense to recurrence formula $P^{m} . \delta^{(k)}(P)-C m, k \delta^{(k-m)}(P)=0$ if $k \geq m$ (see formula 15) considering the condition $\operatorname{grad} P \neq 0$, where the constant $C m, k$ was defined by formula 16 . In the second paragraph we gave a sense to the same formua for the special case $P=P(x)=P\left(x_{1}, \ldots x_{n}\right)=x_{1}^{2}+x_{2}^{2}+\ldots x_{p}^{2}-x_{p+1}^{2}-\ldots x_{p+q}^{2}$.

Our formula is a generalization of the Gelfand and Shilov formula (c.f. ([1]), page 233) and is considered for example, by Bollini, Giambiagi and Tiomno for their theory of analytic regularization in classical Yang-Mills equations and its applications for the singular potentials (c.f. [4]).

Keywords: Recurrence; singular potentials

*Este trabajo es parcialmente soportado por la Comisión de Investigaciones Científicas de la provincia de Buenos Aires (C.I.C.), Argentina 


\section{INTRODUCCIÓN}

De

$$
P\left(x_{1}, \ldots x_{n}\right)=0
$$

La forma $w$ es definida por

$$
d P . w=d v
$$

donde,

$$
d v=d x_{1} \ldots d x_{n}
$$

y $P$ es una función infinitamente diferenciable tal que gradiente de $P=\operatorname{grad} P=\left(\frac{\partial P}{\partial x_{1}}, \frac{\partial P}{\partial x_{2}}, \ldots \frac{\partial P}{\partial x_{n}}\right)$ no se anule en $S$ (lo cual significa que no tiene puntos singulares).

De ([1], página 220, fórmula 2), se tiene

$$
w=(-1)^{j-1} D\left(\begin{array}{l}
x \\
u
\end{array}\right) d u_{1} \ldots d u_{j-1} d u_{j+1} \ldots d u_{n}
$$

donde $D\left(\begin{array}{l}x \\ u\end{array}\right)$ es el Jacobiano de la transformación de $x_{i}$ a $u_{i}$ para $i=1,2, \ldots n$, cual es positivo.

Si en el entorno de un punto dado $\frac{\partial P}{\partial x_{j}} \neq 0$, y seleccionamos las coordenadas de $u_{i}$ de la siguiente forma

$$
\begin{array}{cc}
u_{1}= & x_{1} \\
u_{2}= & x_{2} \\
\cdot & \\
\cdot & \\
\cdot & \\
u_{j}= & P\left(x_{1}, \ldots x_{n}\right) \\
u_{j+1}= & x_{j+1} \\
\cdot & \\
\cdot & \\
\cdot & \\
u_{n}= & x_{n}
\end{array}
$$

entonces

$$
D\left(\begin{array}{l}
x \\
u
\end{array}\right)=\frac{1}{\frac{\partial P}{\partial x_{j}}}
$$

con

$$
\frac{\partial P}{\partial x_{j}}>0
$$

y la forma $w$ definida por (4) se transforma en,

$$
w=\frac{(-1)^{j-1} d x_{1} \ldots d x_{j-1} d x_{j+1} \ldots d x_{n}}{\frac{\partial P}{\partial x_{j}}} .
$$

Por otra parte, de ([1], página 228), se tiene

$$
w_{k}(\varphi)=\frac{\partial^{k}}{\partial u_{1}^{k}}\left\{\varphi_{1} D\left(\begin{array}{l}
x \\
u
\end{array}\right)\right\} d u_{2} \ldots d u_{n}
$$


donde

$$
\varphi_{1}\left(u_{1}, \ldots u_{n}\right)=\varphi\left(x_{1}, \ldots x_{n}\right)
$$

y

$$
w_{o}(\varphi)=\varphi \cdot w
$$

Considerando (9) en ([1], página 230) $\delta^{(k)}(P)$ es definida por

$$
\left\langle\delta^{(k)}(P), \varphi\right\rangle=(-1)^{k} \int_{P=0} w_{k}(\varphi)
$$

También de ([1], página 221, fórmula 8), se tiene

$$
\left\langle\delta^{(k)}(P), \varphi\right\rangle=(-1)^{k} \int_{P=0} \psi_{u_{1}}^{(k)}\left(o, u_{2}, \ldots u_{n}\right) d u_{2} \ldots d u_{n}
$$

donde

$$
\psi_{u_{1}}\left(u_{1}, u_{2}, \ldots u_{n}\right)=\varphi_{1}\left(u_{1}, \ldots u_{n}\right) D\left(\begin{array}{l}
x \\
u
\end{array}\right)
$$

y $D\left(\begin{array}{l}x \\ u\end{array}\right)$ es el Jacobiano de la transformación de $x_{i}$ a $u_{i}$.

En este artículo se le da un sentido a la fórmula de recurrencia $P^{m} \cdot \delta^{(k)}(P)-C m, k \delta^{(k-m)}(P)=0$ si $k \geq m$ (ver 15) considerando la condición $\operatorname{grad} P \neq 0$, donde la constante $C m, k$ es definida por (16). En el segundo parágrafo se le da un sentido a la misma fórmula pero para un caso especial: $P=P(x)=P\left(x_{1}, \ldots x_{n}\right)=x_{1}^{2}+x_{2}^{2}+\ldots x_{p}^{2}-x_{p+1}^{2}-\ldots x_{p+q}^{2}$.

La fórmula que se obtiene es una generalización de fórmulas que aparecen en el libro de Gelfand and Shilov formula (c.f. ([1]), página 233) y es considerada por ejemplo por Bollini, Giambiagi and Tiomno para la teoría de regularización analítica en las ecuaciones clásicas de Yang-Mills y sus aplicaciones para el potencial singular (c.f. [4]).

\section{EL PRODUCTO DE $P^{m} . \delta^{(k)}(P)$}

En este parágrafo, se le da un sentido al producto distribucional $P^{m} \cdot \delta^{(k)}(P)$ donde $P$ es una función infinitamente diferenciable tal que gradiente de $P=\operatorname{grad} P \neq 0$ en $S$ donde $S$ es una hipersuperficie dada por $P\left(x_{1}, \ldots x_{n}\right)=0, m$ es un número entero no-negativo y $\delta^{(k)}(P)$ es la derivada de orden $k$ de la delta de Dirac soportada en $P$ definida por (12).

Teorema 1 Sea $P\left(x_{1}, \ldots x_{n}\right)$ cualquier función suficientemente suave tal que en $P\left(x_{1}, \ldots x_{n}\right)=0, \operatorname{gradP}\left(x_{1}, \ldots x_{n}\right) \neq 0$ (lo cual significa que no hay puntos singulares en $P\left(x_{1}, \ldots x_{n}\right)=0$ ), entonces se tiene la siguiente fórmulas:

$$
P^{m} \cdot \delta^{(k)}(P)=\left\{\begin{array}{cc}
C_{m, k} \delta^{(k-m)}(P) & \text { if } k \geq m \\
y & \\
0 & \text { if } k<m
\end{array}\right.
$$

donde

$$
C_{m, k}=\left(\begin{array}{c}
k \\
m
\end{array}\right) m !(-1)^{m},
$$

$m y k$ son enteros no-negativos, $\delta^{(k)}(P)$ es definida por (12) y $\left(\begin{array}{c}k \\ m\end{array}\right)=\frac{k !}{m !(k-m) !}$.

Demostración. De ([2], página 117) se tiene la siguiente definición:

$$
\langle\alpha . T, \varphi\rangle=\langle T, \alpha . \varphi\rangle
$$




\section{Aguirre}

donde $\varphi \in D, \alpha(x) \in E$ ([2], página 88) y $T \in D^{`}$ (espacio de distribuciones ([2], página 71)).

De (17) y considerando (9) y (12) se tiene,

$$
\left\langle P^{m} \cdot \delta^{(k)}(P), \varphi\right\rangle=\left\langle\delta^{(k)}(P), P^{m} \cdot \varphi\right\rangle=(-1)^{k} \int_{P=0} w_{k}\left(P^{m} . \varphi\right)
$$

donde

$$
w_{k}\left(P^{m} \varphi\right)=\left[\frac{\partial^{k}}{\partial u_{1}^{k}}\left\{u_{1}^{m} \varphi_{1} D\left(\begin{array}{l}
x \\
u
\end{array}\right)\right\}\right]_{u_{1=0}} d u_{2} \ldots d u_{n}
$$

De (19) y considerando la validez de la fórmula de Leibniz para la derivación de un producto se tiene:

Para cada entero positivo $k$,

$$
\begin{gathered}
w_{k}\left(P^{m} \varphi\right)=\left[\sum_{v=o}^{k}\left(\begin{array}{l}
k \\
v
\end{array}\right) \frac{\partial^{k}}{\partial u_{1}^{k}}\left(u_{1}^{m}\right) \frac{\partial^{k-v}}{\partial u_{1}^{k-v}}\left(\varphi_{1} D\left(\begin{array}{l}
x \\
u
\end{array}\right)\right)\right]_{u_{1=0}} d u_{2} \ldots d u_{n}= \\
==\left\{\begin{array}{cc}
0 & \text { if } k<m \\
\text { and } & \text { if } k \geq m .
\end{array}\right.
\end{gathered}
$$

De (18) y considerando (9), (12) y (20) se tiene

$$
P^{m} . \delta^{(k)}(P)=0 \text { si } k<m
$$

y

$$
\begin{gathered}
\left\langle P^{m} . \delta^{(k)}(P), \varphi\right\rangle=(-1)^{k} \int_{P=0}\left(\begin{array}{l}
k \\
m
\end{array}\right) m !\left[\frac{\partial^{k-m}}{\partial u_{1}^{k-m}}\left(\varphi_{1} D\left(\begin{array}{l}
x \\
u
\end{array}\right)\right)\right]_{u_{1=0}} d u_{2} \ldots d u_{n}= \\
=(-1)^{k}\left(\begin{array}{l}
k \\
m
\end{array}\right) m !(-1)^{k-m}\left\langle\delta^{(k-m)}(P), \varphi\right\rangle \text { si } k \geq m
\end{gathered}
$$

De (21) y (22) se obtiene la siguiente fórmula

$$
P^{m} . \delta^{(k)}(P)=\left\{\begin{array}{cc}
\frac{(-1)^{m} k !}{(k-m) !} \delta^{(k)}(P) & \text { if } k \geq m \\
\text { and } & \\
0 & \text { if } k<m .
\end{array}\right.
$$

la cual coincide con (15). El producto (15) puede ser escrito a través de la siguiente fórmula de recurrencia $P^{m} . \delta^{(k)}(P)+\frac{(-1)^{m} k !}{(k-m) !} \delta^{(k)}(P)=0$ si $k \geq m$.

La fórmula (15) es una extensión multidimensional de la fórmula $t^{n} . \delta^{(m)}(t)$ dada en ([3], página 55, sección 2.4, problema 10).

La fórmula (15) es una generalización de la fórmula (6) ([1], página 233) la cual aparece mencionada en el Apéndice A de ([4], fórmula 9A). 


\section{Aguirre}

En efecto, haciendo $m=1$ en (15) y considerando (16) se tiene

$$
P . \delta^{(k)}(P)+k \cdot \delta^{(k-1)}(P)=0,
$$

La fórmula (24) coincide con la fórmula (6) de ([1], página 233) y aparece mencionada en el Apéndice A de ([4], fórmula 9A).

En la próxima sección vamos a estudiar el producto

$$
P^{m} \cdot \delta^{(k)}(P)
$$

Para el caso especial

$$
P=P(x)=P\left(x_{1}, \ldots x_{n}\right)=x_{1}^{2}+x_{2}^{2}+\ldots x_{p}^{2}-x_{p+1}^{2}-\ldots x_{p+q}^{2}
$$

donde $p+q=n$ es la dimensión del espacio.

\section{El Producto singular $P^{m} \cdot \delta^{(k)}(P)$ donde $P$ es definida por (26).}

En este parágrafo, se le da un sentido al producto singular $P^{m} \cdot \delta^{(k)}(P)$ para el caso especial donde $P=P(x)$ es definida por (26).

La superficie dada por la ecuación

$$
x_{1}^{2}+x_{2}^{2}+\ldots x_{p}^{2}-x_{p+1}^{2}-\ldots x_{p+q}^{2}=0
$$

define un hipercono con un punto singular (el vértice) en el origen.

De ([1], páginas 248-249), seleccionando $u_{i}$ de una manera especial y considerando (5) y (13) se tiene la siguiente fórmula:

$$
\left\langle\delta^{(k)}(P), \varphi\right\rangle=(-1)^{k} \int\left[\frac{\partial^{k}}{\partial P^{k}}\left\{\frac{1}{2} \varphi\left(r^{2}-P\right)^{\frac{q-2}{2}}\right\}\right]_{P=0} r^{p-1} d \Omega_{p} d \Omega_{q} d r
$$

donde

$$
\begin{gathered}
r=\sqrt[2]{x_{1}^{2}+x_{2}^{2}+\ldots x_{p}^{2},} \\
s=\sqrt[2]{x_{p+1}^{2}+\ldots+x_{p+q}^{2}},
\end{gathered}
$$

$d \Omega_{p}$ y $d \Omega_{q}$ son elementos de área de superficie de las esferas unitaria en $R^{p}$ y $R^{q}$ respectivamente.

Haciendo

$$
\psi(r, s)=\int \varphi d \Omega_{p} d \Omega_{q}
$$

De (28) se tiene

$$
\left\langle\delta^{(k)}(P), \varphi\right\rangle=\int_{o}^{\infty}\left[\left(\frac{\partial}{2 s \partial s}\right)^{k}\left\{s^{q-2} \frac{\psi(r, s)}{2}\right\}\right]_{s=r} r^{p-1} d r
$$

y

$$
\left\langle\delta^{(k)}(P), \varphi\right\rangle=(-1)^{k} \int_{o}^{\infty}\left[\left(\frac{\partial}{2 r \partial r}\right)^{k}\left\{r^{p-2} \frac{\psi(r, s)}{2}\right\}\right]_{r=s} s^{q-1} d r
$$

([1], página 249). 
En ([1], página 249), Gelfand and Shilov han usado (32) y (33) como la definición de la distribución $\delta^{(k)}(P)$ bajo la condición

$$
k<\frac{p+q-2}{2}
$$

Por tanto, las integrales en (32) y (33) convergen bajo la condición (34).

$\mathrm{Si}$, por otra parte

$$
k \geq \frac{p+q-2}{2}
$$

se debería entender la definición de $\left\langle\delta_{1}^{(k)}(P), \varphi\right\rangle$ y $\left\langle\delta_{2}^{(k)}(P), \varphi\right\rangle$ como la regularización de (35) y respectivamente([1], páginas 249-250).

Bajo la misma condición y haciendo uso del mismo método ([1], páginas 248-250), se le da un sentido a la misma fórmula de recurrencia obtenida en (15) pero ahora para el caso especial

$P=P(x)=P\left(x_{1}, \ldots x_{n}\right)=x_{1}^{2}+x_{2}^{2}+\ldots x_{p}^{2}-x_{p+1}^{2}-\ldots x_{p+q}^{2}$.

Teorema 2: Sea $P=P(x)$ definida por (26), $m$ entero no-negativo y $\delta^{(k)}(P)$ definida por la ecuación (28), entonces la siguiente fórmula es válida

$$
P^{m} . \delta^{(k)}(P)=\left\{\begin{array}{cc}
\frac{(-1)^{m} k !}{(k-m) !} \delta^{(k-m)}(P) & \text { if } k \geq m \\
\text { and } & \\
0 & \text { if } k<m
\end{array}\right.
$$

bajo la condición $k<\frac{p+q-2}{2}$. Donde $p+q=n$ es la dimensión del espacio.

Demostración. De (18) y considerando (28) y (32) se tiene

$$
\begin{gathered}
\left\langle P^{m} \cdot \delta^{(k)}(P), \varphi=\right. \\
=(-1)^{k} \int\left[\frac{\partial^{k}}{\partial P^{k}}\left\{P^{m} \cdot \frac{1}{2} \varphi\left(r^{2}-P\right)^{\frac{q-2}{2}}\right\}\right]_{P=0} r^{p-1} d \Omega_{p} d \Omega_{q} d r= \\
=\int_{o}^{\infty}\left[\left(\frac{\partial}{2 s \partial s}\right)^{k}\left\{\left(r^{2}-s^{2}\right)^{m} s^{q-2} \frac{\psi(r, s)}{2}\right\}\right]_{s=r} r^{p-1} d r
\end{gathered}
$$

si $k<\frac{p+q-2}{2}$.

Por otra parte, haciendo el cambio formal de variables

$$
\begin{aligned}
& u=r^{2} \\
& v=s^{2}
\end{aligned}
$$

En (32) y escribiendo

$$
\psi(r, s)=\psi_{1}(u, v)
$$

se tiene

$$
\left\langle\delta^{(k)}(P), \varphi\right\rangle=\frac{1}{4} \int_{o}^{\infty}\left[\frac{\partial^{k}}{\partial v^{k}}\left\{v^{\frac{q-2}{2}} \psi_{1}(u, v)\right\}\right]_{v=u} u^{\frac{p-2}{2}} d u
$$

([1], página 250). 
De (37) y considerando (38) y (39) se tiene,

$$
\begin{gathered}
\left\langle P^{m} \cdot \delta^{(k)}(P), \varphi\right\rangle= \\
=\frac{1}{4} \int_{o}^{\infty}\left[\frac{\partial^{k}}{\partial v^{k}}\left\{(u-v)^{m} v^{\frac{q-2}{2}} \psi_{1}(u, v)\right\}\right]_{v=u} u^{\frac{p-2}{2}} d u
\end{gathered}
$$

si $k<\frac{p+q-2}{2}$.

Ahora considerando la fórmula de Leibniz para la derivación de un producto se tiene:

$$
\begin{gathered}
{\left[\frac{\partial^{k}}{\partial v^{k}}\left\{(u-v)^{m} v^{\frac{q-2}{2}} \psi_{1}(u, v)\right\}\right]_{v=u}=} \\
=\left\{\begin{array}{cc}
\frac{k !(-1)^{m}}{(k-m) !}\left[\frac{\partial^{k}-m}{\partial v^{k-m}}\left\{v^{\frac{q-2}{2}} \psi_{1}(u, v)\right\}\right]_{v=u} & \text { si } k \geq m \\
0 & \text { si } k<m .
\end{array}\right.
\end{gathered}
$$

De (41) y (42) se tiene

$$
\left\langle P^{m} \cdot \delta^{(k)}(P), \varphi\right\rangle=\begin{array}{cc}
\left.\frac{(-1)^{m} k !}{(k-m) !} \delta^{(k-m)}(P), \varphi\right\rangle & \text { si } k \geq m \\
y & \text { si } k<m
\end{array}
$$

y $k<\frac{p+q-2}{2}$.

De (43) se obtiene la siguiente fórmula:

$$
P^{m} . \delta^{(k)}(P)=\left\{\begin{array}{cc}
\frac{(-1)^{m} k !}{(k-m) !} \delta^{(k-m)}(P) & \text { si } k \geq m \\
\text { and } & \\
0 & \text { si } k<m
\end{array}\right.
$$

y $k<\frac{p+q-2}{2}$, la cual coincide con (36). El producto (36) puede ser escrito a través de la siguiente fórmula de recurrencia $P^{m} \cdot \delta^{(k)}(P)-\frac{(-1)^{m} k !}{(k-m) !} \delta^{(k)}(P)=0$ si $k \geq m$ y $k<\frac{n}{2}-1$. Donde $P=P(x)=P\left(x_{1}, \ldots x_{n}\right)=x_{1}^{2}+x_{2}^{2}+\ldots x_{p}^{2}-x_{p+1}^{2}-\ldots x_{p+q}^{2}$. Cuando $m=k$ la fórmula (36) aparece en ([5]) la cual fue probada por un método completamente diferente del que se presenta en este artículo. 


\section{REFERENCIAS}

[1] I.M.Gelfand and G.L. Shilov., Generalized functions, Vol. I, Academic Press, New York, 1964.

[2] L.Schwartz., Thèorie des distributions, Hermann, Paris, 1966.

[3] A.H.Zemanian., Distributions theory and Transform Analysis, International series in Pure and Applied Mathematics, McGraw-Hill Books Company, 1965.

[4] C.G.Bollini, J. J. Giambiagi and J. Tiomno., Singular potentials and analytic regularization in classical Yang-Mills equations, J. Math. Phys, 20 (9), september 1979.

[5] S.E.Trione., Products between $\delta^{(k)}\left(m^{2}+P\right)$ and the distributions $\left(m^{2}+P\right)^{k}$, Preprint Nro.55, Instituto Argentino de Matemática, IAM-CONICET, Buenos Aires Argentina, 1983.

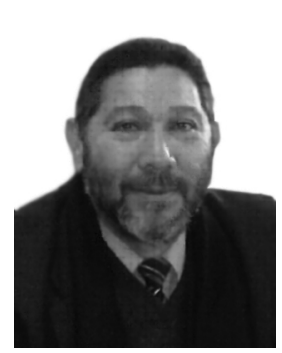

Manuel A. Aguirre, es Profesor y Decano de la Facultad de Ciencias Exactas de la Universidad Nacional del Centro de la Provincia de Buenos Aires, Núcleo Consolidado Matemática Pura y Aplicada-NUCOMPA

Facultad de Ciencias Exactas UNCentro

Paraje Arroyo Seco, 7000-Tandil

Provincia de Buenos Aires, Argentina

Tel.: +54 2293439657

E-mail: maguirre@exa.unicen.edu.ar 